\title{
Comparisons of MOSFET and Relay Switches in Impedance Matching Networks for Wireless Power Transfer
}

\author{
Cristina A. Alexandru \\ College of Engineering, Mathematics and Pysical Sciences \\ University of Exeter \\ Exeter, UK \\ ca410@exeter.ac.uk
}

\author{
Dibin Zhu \\ College of Engineering, Mathematics and Physical Sciences \\ University of Exeter \\ Exeter, UK \\ d.zhu@exeter.ac.uk
}

\begin{abstract}
This paper presents the comparison between using MOSFET and relay switches in impedance matching networks (IMN) for inductive wireless power transfer (WPT). Complex IMN can be designed with multiple tuning branches which match the system with the optimum impedance. In such cases, it is required for the branches to be turned on and off accordingly. This is achieved through the use of switches. Existing research have mainly used electromechanical relays to perform the switching. However, relays have the disadvantage of increasing the IMN size, especially in complex designs. As an alternative, the MOSFET can be implemented as a switch with a smaller footprint. It is concluded that the MOSFET AC switch is a suitable option instead of relays but it requires careful consideration when implemented due to its parasitic elements.
\end{abstract}

Keywords- Impedance Matching Network, MOSFET switch, Relay, Wireless Power Transfer

\section{INTRODUCTION}

Misalignment and separation between the transmitter and receiver coils in wireless power transfer (WPT) cause variations in the optimum transmitter and receiver impedance [1]. The variation in impedance leads to drops in the power transfer efficiency. Such losses can be compensated by introducing impedance matching networks (IMN) in the system [1]-[2]. IMN matches the system to the optimum impedance by selecting the corresponding tuning capacitor. This is achieved through the use of switches.

In existing research, mechanical switches and relays have mainly been implemented to perform the switching. For example, research studies in [1] present an array of parallel capacitors where each branch is connected to a switch, with the purpose of achieving maximum efficiency for multiple load devices. The switches used are a slide-style dual in-line package and switching is achieved manually. Designs such as [3]-[4] have focused on creating an automated algorithm to pair the most suitable capacitor to the network through the use of an electromechanical relay (EMR) switch (Panasonic ARE10A06).

In complex designs constituting of multiple capacitors using EMR switches offer the disadvantage of an increased IMN size if compared to MOSFET switches or a non-switchable IMN [5]. Therefore, the MOSFET switch is an alternative which currently in literature has not been thoroughly investigated for this application. Furthermore, the configuration of the switches and the effect caused by parasitic elements also need further investigation.

In this paper, a parallel-series (PS) IMN consisting of two parallel branches is implemented at the receiver of a WPT system. On both branches, both MOSFET and EMR switches have been implemented with the purpose of comparing their performance. Section II presents the WPT system and MOSFET model. Section III covers the experimental analysis. Lastly, section IV discusses the experimental results and provides a conclusion.

\section{WPT SYSTEM MODEL}

\section{A. WPT Circuit Model}

The equivalent circuit of the WPT system proposed includes a dual switchable PS capacitive IMN on the receiver, as shown in Fig.1. The impedance network constitutes of three tuning capacitors, in which $\mathrm{C}_{\mathrm{Rx} 2}$ and $\mathrm{C}_{\mathrm{Rx} 3}$ represent the two switchable parallel branches.

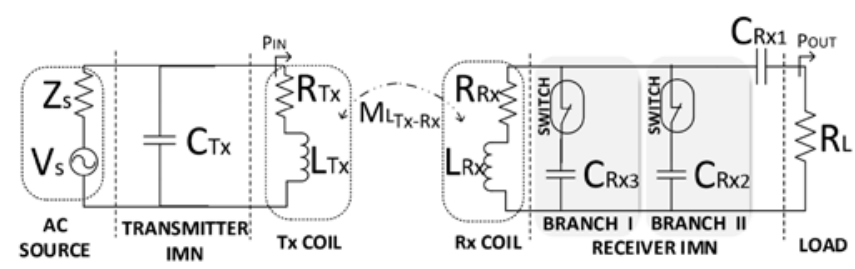

Fig. 1. WPT system equivalent model - Transmitter, Tx with a dual switchable PS-IMN at the receiver, $\mathrm{Rx}$

\section{B. MOSFET Switch Model}

The MOSFET AC switch constitutes of two n-type MOSFETs which are connected as shown in Fig. 2(a) with a DC voltage controlling both gate terminals whilst the source terminals are grounded. The two drains represent the two terminals of the AC switch.

To turn on the switch, a DC voltage greater than the threshold voltage, $\mathrm{V}_{\mathrm{TH}}$, of the MOSFET is applied across the gate and the source terminals. A voltage lower than $\mathrm{V}_{\mathrm{TH}}$ will turn off the switch. Fig. 2(b) illustrates the switch equivalent model which is represented by its parasitic resistor, $R_{p}$ and capacitor, $C_{p}$ connected in parallel [5]. 


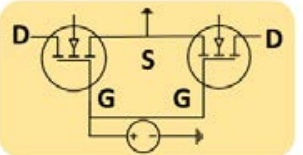

a)

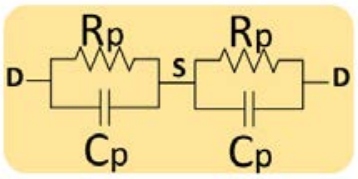

b)
Fig. 2. MOSFET AC switch circuit connections (a) and equivalent model (b)

Based on this model, the equivalent impedance formula of a MOSFET AC switch is derived in Eq. (1).

$$
\mathrm{Z}_{\text {switch }}=\frac{2 * \mathrm{Z}_{\mathrm{Rp}} * \mathrm{Z}_{\mathrm{Cp}}}{\mathrm{Z}_{\mathrm{Rp}}+\mathrm{Z}_{\mathrm{Cp}}}
$$

where $\mathrm{Z}_{\mathrm{Cp}}=\frac{1}{\mathrm{j} \omega \mathrm{Cp}}, \mathrm{Z}_{\mathrm{Rp}}=\mathrm{R}_{\mathrm{p}}$.

\section{EMR Relay Switch Model}

The relay switch is characterised by a contact resistance when turned on. For this application, the chosen EMR relay (OZ-SS-112LM1) has an on-resistance of $0.1 \Omega$.

\section{WPT System Configuration}

Based on the circuit model in Fig. 1 and taking into consideration the MOSFET AC switch equivalent circuit from Fig. 2(b), a mathematical model was developed.

Using the mathematical model, the efficiency of the WPT system was analysed for two scenarios, i.e. when the switch is on and off. It was found that when the switch is on, a small onresistance is desirable as it shorts the parasitic capacitor and does not influence the equivalent impedance of the network [5]. In contrast, when the switch is off, the off-resistance is large and can be treated as open circuit. It was concluded that in order to isolate to branch from the circuit, it is preferred for the MOSFET to have a small parasitic capacitance so as not the affect the equivalent impedance of the IMN and thus, the efficiency [5]. Considering that in the experiment, it was infeasible to determine the value of $\mathrm{C}_{\mathrm{p}}$, the MOSFET with low on-resistance was selected.

Fig.3 illustrates the IMN equivalent circuit when only one branch is implemented, Fig. 3(a) and when one of the branches is turned on and the other off, Fig. 3(b). The total impedance equations were derived based on the two circuit models, respectively in Eqs. (2)-(3), where $Z_{\text {switch }}$ has been defined previously in Section II.B. The same principle can be applied to the second case, when branch I is off and branch II is on.

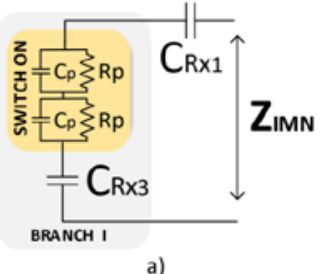

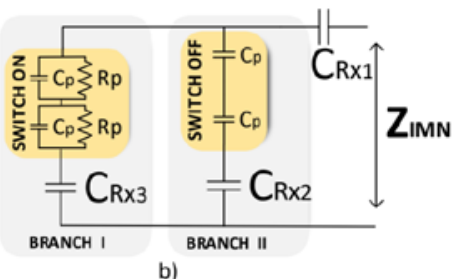

Fig. 3. Receiver IMN at with branch I only (a) and both tuning branches with branch II off (b)

$$
\mathrm{Z}_{\mathrm{IMN}}=\frac{\mathrm{Z}_{\mathrm{CRx} 3 \mathrm{ON}} * \mathrm{Z}_{\mathrm{CRx} 1}}{\mathrm{Z}_{\mathrm{CRx} 3 \mathrm{ON}}+\mathrm{Z}_{\mathrm{CRx} 1}}
$$

where $\mathrm{Z}_{\mathrm{CRx} 3 \text { ON }}=\mathrm{Z}_{\text {switch }}+\mathrm{Z}_{\mathrm{CRx} 3}$.

$$
\mathrm{Z}_{\mathrm{IMN}}=\frac{\mathrm{Z}_{\mathrm{CRx} 3 \mathrm{ON}} * \mathrm{Z}_{\mathrm{CRx} 2 \mathrm{OFF}} * \mathrm{Z}_{\mathrm{CRx} 1}}{\mathrm{Z}_{\mathrm{CR \times 3} \text { ON }} * \mathrm{Z}_{\mathrm{CRx} 2 \mathrm{OFF}}+\mathrm{Z}_{\mathrm{CRx} 2 \mathrm{OFF}} * \mathrm{Z}_{\mathrm{CRx} 1}+\mathrm{Z}_{\mathrm{CRx} 3 \mathrm{ON}} * \mathrm{Z}_{\mathrm{CRx} 1}}
$$

where $\mathrm{Z}_{\mathrm{CRx} 2 \text { ofF }}=2 * \mathrm{Z}_{\mathrm{Cp}}+\mathrm{Z}_{\mathrm{CRx} 2}$.

\section{EXPERIMENTAL ANALYSIS}

\section{A. WPT System Experimental Setup}

The circuit diagram in Fig. 4 illustrates the system's structure. At the transmitter, a PIC18F microcontroller is used to drive the H-bridge inverter at $250 \mathrm{kHz}$ or $200 \mathrm{kHz}$, which is connected to the tuning capacitor, $\mathrm{C}_{\mathrm{Tx}}$ and transmitter coil, $\mathrm{T}_{\mathrm{x}}$. A shunt resistor, $R_{\text {shunt }}$, was introduced in series with the transmitter coil for the measurement of the AC transmitting current. The receiver constitues of the receiver coil, $R_{x}$, IMN and receiver load, $\mathrm{R}_{\mathrm{L}}$.

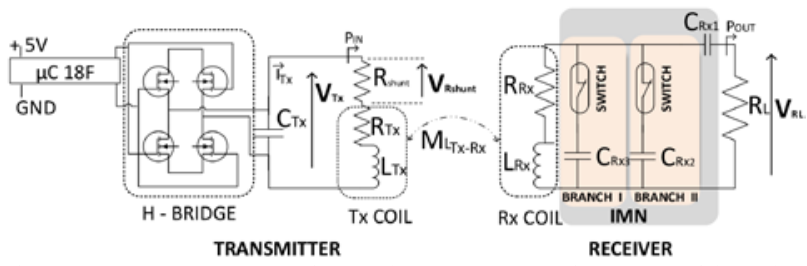

Fig. 4. WPT system experimental setup -Transmitter, Tx with a dual switchable IMN at the receiver, Rx

In this model, the IMN was designed to accommodate two operating frequencies, where $\mathrm{C}_{\mathrm{Rx} 1}$ is a fixed series capacitor always connected to the load. Branch I integrates in the receiver circuit, $\mathrm{C}_{\mathrm{Rx} 2}$ which corresponds to the tuning capacitor for an operating frequency of $250 \mathrm{kHz}$. Similarly, $\mathrm{C}_{\mathrm{Rx} 3}$ located in branch II corresponds to a frequency of $200 \mathrm{kHz}$. Depending on which frequency is selected, the corresponding branch is turned
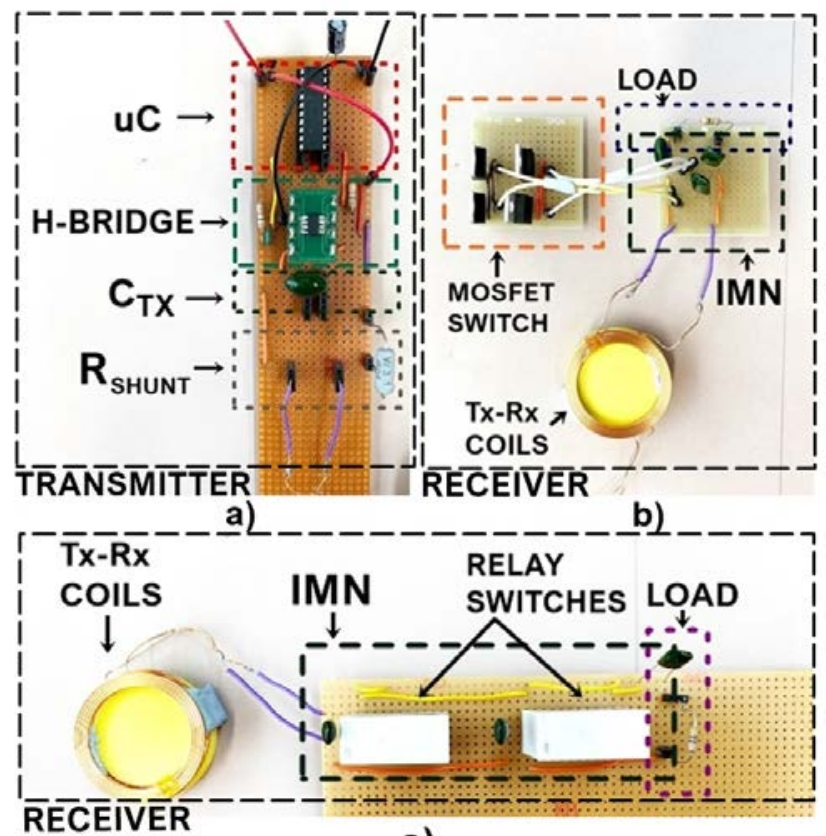

c)

Fig. 5. WPT experimental setup - Transmitter, Tx (a) and switchable MOSFET (b) and Relay (c) IMN at the receiver, Rx 
on and the other off through the use of switches. Fig. 5 shows the test setup with the two switching options for the IMN, the MOSFET switch and relay. The transmitter and receiver coils are perfectly aligned and $5 \mathrm{~mm}$ apart.

Firstly, for each operating frequency, the optimum tuning capacitor values for both the transmitter and the receiver have been established in the absence of switches as listed in Table I. All parameters in Table I have been tested and measured experimentally for the transmitter and receiver circuits.

TABLE I

WPT SYSTEM EXPERIMENTAL AND SIMULATED PARAMETERS

\begin{tabular}{lcr}
\hline \hline Symbol & Quantity & \multicolumn{1}{c}{ Value } \\
\hline $\mathrm{L}_{\mathrm{Tx}}$ & Transmitter coil inductance & $30 \mu \mathrm{H}$ \\
$\mathrm{L}_{\mathrm{Rx}}$ & Receiver coil inductance & $30 \mu \mathrm{H}$ \\
$\mathrm{M}_{\mathrm{LTx}-\mathrm{Rx}}$ & Mutual inductance & $14.4 \mu \mathrm{H}$ \\
$\mathrm{C}_{\mathrm{Tx}}$ & Transmitter tuning capacitor at $250 \mathrm{kHz}$ & $33 \mathrm{nF}$ \\
$\mathrm{C}_{\mathrm{Tx}}$ & Transmitter tuning capacitor at $200 \mathrm{kHz}$ & $22 \mathrm{nF}$ \\
$\mathrm{C}_{\mathrm{Rx} 1}$ & Receiver series tuning capacitor & $12 \mathrm{nF}$ \\
$\mathrm{C}_{\mathrm{Rx} 2}$ & Receiver switchable parallel tuning capacitor & $14.7 \mathrm{nF}$ \\
$\mathrm{C}_{\mathrm{R} 3}$ & Receiver switchable parallel tuning capacitor & $5.6 \mathrm{nF}$ \\
\hline
\end{tabular}

Secondly, the power transfer efficiency was calculated using the efficiency formula in Eq. (4), where the input and output power was calculated by measuring the transmitter coil RMS voltage, $\mathrm{V}_{\mathrm{Tx}}$, transmitter RMS current, $\mathrm{I}_{\mathrm{Tx}}$, and the RMS voltage across the variable load, $\mathrm{V}_{\mathrm{RL}}$.

$$
\eta=\frac{P_{O U T}}{P_{I N}}=\frac{\frac{v_{R L}{ }^{2}}{R_{L}}}{v_{T X} I_{T X}}
$$

The efficiency is calculated under the same conditions for various load resistances and the same method is used when the switches are connected on the IMN parallel branches. All the results presented are normalised to the maximum efficiency at the optimum load resistance when no switch is used.

\section{B. Analysis of the adaptive IMN - using MOSFET switches}

From the theoretical analysis of the switch mentioned in Section II.D, it is known that the on-resistance of the MOSFET has an important role. Previous study [5] showed that the CSD18533KCS MOSFET has minimum effect on the WPT efficiency due to its low on-resistance of $5 \mathrm{~m} \Omega$. This MOSFET is also characterized by a parasitic capacitance of $2420 \mathrm{pF}$. Ideally, for this application the parasitic capacitance should be as small as possible.

At the operating frequency of $250 \mathrm{kHz}$, the corresponding branch, branch I is integrated in the circuit by turning on the MOSFET switch and the latter, branch II is disconnected by turning off the switch. Consequently, the same methodology is applied when the system frequency changes to $200 \mathrm{kHz}$.

\section{Scenario $1-250 \mathrm{kHz}$}

Firstly, at the operating frequency of $250 \mathrm{kHz}$, branch I is turned on and branch II is disconnected by turning off the MOSFET switch. The results presented in Fig. 6 and Table II indicate that at the optimum load value, this configuration results in lower peak efficiency compared to the case without the branch II implementation.

Considering that turning off the MOSFET switch in branch II introduces in the IMN an unwanted impedance, it needs to adjust the value of the tuning capacitor in Branch $\mathrm{I}$ in order to compensate for the efficiency drop. For the circuit configuration shown in Fig. 3(a), $\mathrm{Z}_{\mathrm{IMN}}$ is $45.2 \Omega$ and for Fig. 3(b) is $42.5 \Omega$. To compensate for the difference between the two impedances, the adjusted value of the tuning capacitor, $\mathrm{C}_{\mathrm{Rx} 3}$ is set from 5.6 $\mathrm{nF}$ to $3.9 \mathrm{nF}$.

When $\mathrm{C}_{\mathrm{Rx} 3}$ is set at $3.9 \mathrm{nF}$, the maximum efficiency can be achieved under the same optimum load. It is also important to notice that optimum load value when $\mathrm{C}_{\mathrm{Rx} 3}$ is not adjusted changes to $12 \Omega$, indicating that a change occurred in the equivalent impedance of the IMN.

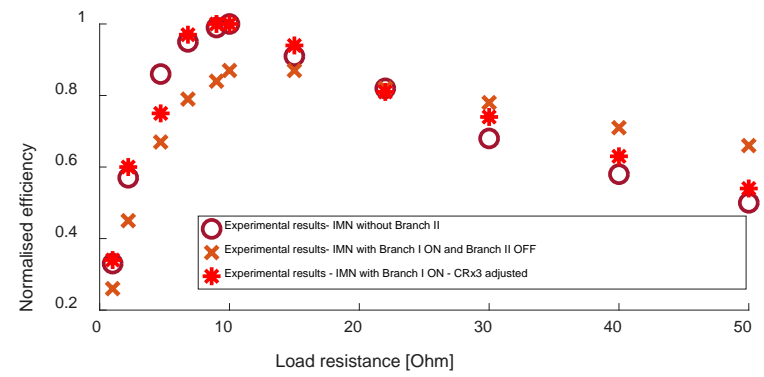

Fig. 6. Comparison of experimental results - Normalised efficiency vs load resistance - model operating at $250 \mathrm{kHz}$ with MOSFET switch

TABLE II

WPT SYSTEM EXPERIMENTAL PARAMETERS - OPERATING AT $250 \mathrm{KHZ}$ USING MOSFET SWITCHES

\begin{tabular}{lcc}
\hline \hline IMN Configuration & Max. Efficiency & Optimum $\mathrm{R}_{\mathrm{L}}[\Omega]$ \\
\hline IMN without Branch II & 1.00 & 9 \\
Branch I ON -Branch II OFF & 0.90 & 12 \\
Branch I ON - C $\mathrm{Rx3}_{\text {adjusted }}$ adje & 1.00 & 9 \\
\hline \hline
\end{tabular}

\section{Scenario $2-200 \mathrm{kHz}$}

Secondly, at the operating frequency of $200 \mathrm{kHz}$, branch I is turned off and branch II is on. Using as a model the circuit configuration from Fig.3 and Eqs. (2)-(3), the impedance equations were derived on the same principle. At this stage, the value of $\mathrm{C}_{\mathrm{Rx} 3}$ is set to $3.9 \mathrm{nF}$ matching the optimum tuning value. Turning off the MOSFET switch from branch I added to

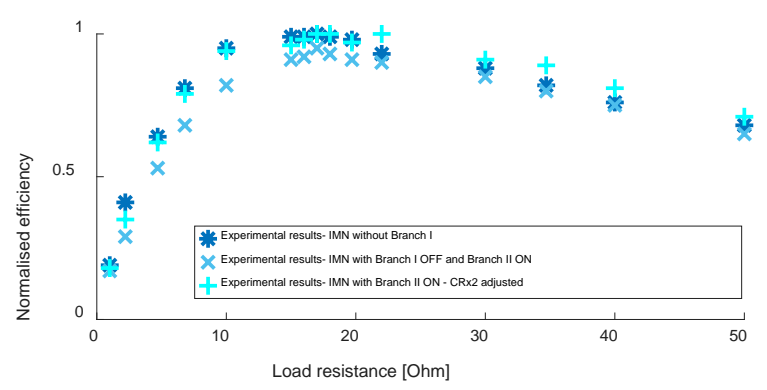

Fig. 7. Comparison of experimental results - Normalised efficiency vs load resistance - model operating at $200 \mathrm{kHz}$ with MOSFET switch 
the IMN an unwanted impedance, shifting the optimum $\mathrm{Z}_{\text {IMN }}$ of $29.8 \Omega$ to $28.8 \Omega$. Therefore, the value of the tuning capacitor, $\mathrm{C}_{\mathrm{Rx} 2}$ was adjusted from the original value of $14.7 \mathrm{nF}$ to $13 \mathrm{nF}$, which allowed the efficiency to increase at the maximum value.

Considering this MOSFET behaviour is observed for both operating frequencies, it concludes that designing a complex IMN consisting of multiple MOSFET switches, requires careful attention.

TABLE III

WPT SYSTEM EXPERIMENTAL PARAMETERS - OPERATING AT $200 \mathrm{KHZ}$ USING MOSFET SWITCHES

\begin{tabular}{lcc}
\hline \hline IMN Configuration & Max. Efficiency & Optimum $\mathrm{R}_{\mathrm{L}}[\Omega]$ \\
\hline IMN without Branch I & 1.00 & 18 \\
Branch I OFF - Branch II ON & 0.94 & 17 \\
Branch II ON - $\mathrm{C}_{\mathrm{Rx} 2}$ adjusted & 1.00 & 18 \\
\hline \hline
\end{tabular}

\section{Analysis of the adaptive IMN - using Relays}

The same approach mentioned in Section III.C was followed when implementing the EMR relay (OZ-SS-112LM1) as the switching element in the IMN.

\section{Scenario $1-250 \mathrm{kHz}$}

Analysing the results from Fig.8 and Table IV, it is observed that when Branch I is turned on and the latter is turned off, the relay does not cause any negative effect to the efficiency, having the same response as the case when no switch is integrated.

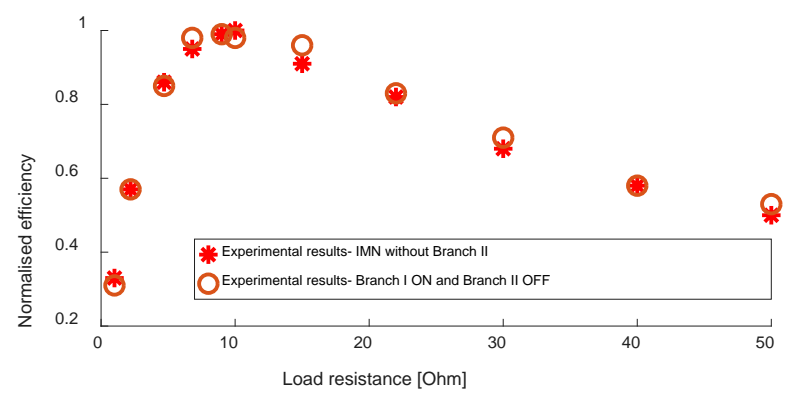

Fig. 8. Comparison of experimental results - Normalised efficiency vs load resistance - model operating at $250 \mathrm{kHz}$ with Relay

TABLE IV

WPT SYSTEM EXPERIMENTAL PARAMETERS - OPERATING AT $250 \mathrm{KHZ}$ USING RELAYS

\begin{tabular}{lcc}
\hline \hline \multicolumn{1}{c}{ IMN Configuration } & Max. Efficiency & Optimum $\mathrm{R}_{\mathrm{L}}[\Omega]$ \\
\hline IMN without Branch II & 1.00 & 9 \\
Branch I ON - Branch II OFF & 0.99 & 9 \\
\hline \hline
\end{tabular}

\section{Scenario 2- $200 \mathrm{kHz}$}

Changing the operating frequency to $200 \mathrm{kHz}$ and turning on branch II, the same response as in Scenario 1 is observed as illustrated in Fig. 9 and Table V. Consequently, it was not necessary to intervene and modify any of the original parameters of the tuning capacitors.

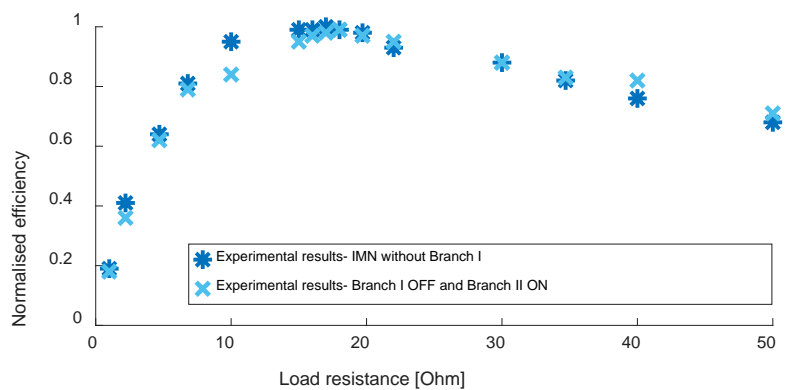

Fig. 9. Comparison of experimental results - Normalised efficiency vs load resistance - model operating at $200 \mathrm{kHz}$ with Relay

TABLE V

WPT SYSTEM EXPERIMENTAL PARAMETERS - OPERATING AT $200 \mathrm{KHZ}$ USING RELAYS

\begin{tabular}{lcc}
\hline \hline \multicolumn{1}{c}{ IMN Configuration } & Max. Efficiency & OptimumR $_{\mathrm{L}}[\Omega]$ \\
\hline IMN without Branch II & 1.00 & 9 \\
Branch I ON - Branch II OFF & 0.99 & 9 \\
\hline
\end{tabular}

\section{CONCLUSION}

It is concluded from the results presented in Section III that the MOSFET with a low on-resistance such as CSD18533KCS is a feasible choice as an alternative to EMR relays in IMN for WPT. The suitable switch for this application is characterised by a low on-resistance so that the parasitic capacitor of the MOSFET switch is short-circuited and the IMN impedance is not affected. According to the results, when the MOSFET is turned off, it still has an impact by introducing in the circuit the parasitic capacitance of switch. Therefore, the parasitic capacitor value should be as small as possible so that when turned off, the switch will not alter the equivalent impedance of the network greatly.

Comparing the two switches, relay switches have the disadvantage of being considerably more space-consuming compared to MOSFETs. For example, each OZ-SS-112LM1 relay switch has a volume of $6.96 \mathrm{~cm}^{3}$. Alternatively, the MOSFET has a volume of $0.576 \mathrm{~cm}^{3}$, offering the advantage of reducing the IMN size. A pair of such MOSFETs have a total volume of $1.152 \mathrm{~cm}^{3}$, which is $83 \%$ smaller than the relay switch. Additionally, terms of price range both switches are within a similar price range. Therefore, AC MOSFET switches are a suitable choice for automated switchable IMN in WPT systems.

\section{REFERENCES}

[1] Kim, J., Kim, D.-H., and Park, Y .-J.: 'Free-Positioning Wireless Power Transfer to Multiple Devices Using a Planar Transmitting Coil and Switchable Impedance Matching Networks', IEEE Transactions on Microwave Theory and Techniques, vol. 64, no. 11, 2016.

[2] K. K. Ean, B. T. Chuan, T. Imura, and Y. Hori, "Impedance matching and power division algorithm considering cross coupling for wireless power transfer via magnetic resonance,” in Proc. IEEE 34th Int. Telecommun. Energy Conf. (INTELEC), Sep. 2012, pp. 1-5. 
[3] L. Yongseok, T. Hoyoung, L. Seungok and P. Jongsun, "An Adaptive Impedance-Matching Network Based on a Novel Capacitor Matrix for Wireless Power Transfer,” Power Electronics, IEEE Transactions on, vol. 29, no. 8, pp. 4403-4413, Aug. 2014
[4] J. Kim and J. Jeong, "Range-Adaptive Wireless Power Transfer using Multi-loop and Tunable Matching Techniques," IEEE Trans. Ind. Electron., vol. 62, no. 10, pp. 6233-6241, Oct. 2015.

[5] C.A Alexandru and D.Zhu, "Exploitation of MOSFET based AC Switches in Capacitive Impedance Matching Networks in Wireless Power Transfer Systems”, under preparation. 